ANNALES

POLONICI MATHEMATICI

$83.1(2004)$

\title{
On the equivalence of Green functions for general Schrödinger operators on a half-space
}

\author{
by AbDoul Ifra and Lotfi Riahi (Tunis)
}

\begin{abstract}
We consider the general Schrödinger operator $L=\operatorname{div}\left(A(x) \nabla_{x}\right)-\mu$ on a half-space in $\mathbb{R}^{n}, n \geq 3$. We prove that the $L$-Green function $G$ exists and is comparable to the Laplace-Green function $G_{\Delta}$ provided that $\mu$ is in some class of signed Radon measures. The result extends the one proved on the half-plane in [9] and covers the case of Schrödinger operators with potentials in the Kato class at infinity $K_{n}^{\infty}$ considered by Zhao and Pinchover. As an application we study the cone $\mathcal{C}_{L}\left(\mathbb{R}_{+}^{n}\right)$ of all positive $L$-solutions continuously vanishing on the boundary $\left\{x_{n}=0\right\}$.
\end{abstract}

1. Introduction In the last two decades, the problem of comparability of Green functions for elliptic operators has been discussed by several authors in different situations. For elliptic operators with sufficiently regular coefficients on bounded smooth domains, we refer the reader to [1] and [4]. In [2], Cranston, Fabes and Zhao studied the problem for Schrödinger operators with potentials in the Kato class $K_{n}^{\text {loc }}$ on bounded Lipschitz domains. Their work extends the one due to Zhao [10]. For Schrödinger operators with short range potentials on Lipschitz domains (bounded or unbounded) with compact boundary, the problem was studied by Herbst and Zhao in [3]. In [6], Pinchover studied the problem for elliptic operators on $\mathbb{R}^{n}$ with lower order terms in the Kato class at infinity, $K_{n}^{\infty}$, which contains the class of short range potentials. However, for Schrödinger operators on arbitrary domains such as unbounded domains with noncompact boundary, nothing is proved about this problem. In this paper we are interested in the problem for the general Schrödinger operator

$$
L=L_{0}-\mu,
$$

where $L_{0}=\operatorname{div}\left(A(x) \nabla_{x}\right)$ on the half-space $\mathbb{R}_{+}^{n}=\left\{x=\left(x_{1}, \ldots, x_{n}\right) \in \mathbb{R}^{n}\right.$ : $\left.x_{n}>0\right\}, n \geq 3$. The matrix $A$ is assumed to be real, symmetric, uniformly elliptic with locally Lipschitz continuous coefficients and $\mu$ is a signed Radon

2000 Mathematics Subject Classification: 31B05, 31B25, 35J60.

Key words and phrases: Green function, positive solution, Schrödinger operator. 
measure. By [8] we know that the $L_{0}$-Green function $G_{0}$ is comparable to the Laplace-Green function $G_{\Delta}$ on $\mathbb{R}_{+}^{n}$.

Our main purpose is to study the existence of the $L$-Green function and its comparability to $G_{\Delta}$ on the half-space $\mathbb{R}_{+}^{n}$ when $\mu$ is in a general class of signed Radon measures denoted by $\mathcal{K}$. The Schrödinger operator $L=L_{0}-V(x)$ with potential $V$ in the class $K_{n}^{\infty}$ introduced by Zhao [11, 12 ] and Pinchover [6] is just the special case when $\mu$ has the density $V$ with respect to the Lebesgue measure. Hence our result extends those proved by Herbst and Zhao [3] and Pinchover [6] to the case of the half-space. The comparability result enables us to obtain potential-theoretic results for $L$ which are known to hold for $\Delta$. We prove as an application a Liouville type theorem for $L$-positive solutions on the half-space $\mathbb{R}_{+}^{n}$.

The paper is organized as follows. In Section 2, we give some preliminaries and notations. In Section 3, we introduce the class $\mathcal{K}$ and we study some of its properties. In particular we prove that this class strictly contains the class of measures $V(x) d x$ with $V$ in $K_{n}^{\infty}$. In Section 4 , we prove the existence of the $L$-Green function and the comparability result when $\mu$ is in $\mathcal{K}$ with minimal condition. In Section 5 , we study the structure of $\mathcal{C}_{L}\left(\mathbb{R}_{+}^{n}\right)$, the cone of positive $L$-solutions on $\mathbb{R}_{+}^{n}$ continuously vanishing on the boundary. We show that $\mathcal{C}_{L}\left(\mathbb{R}_{+}^{n}\right)$ is a one-dimensional cone, a result which fails to hold even in simple cases such as $L=\Delta-c$, with $c \in \mathbb{R} \backslash\{0\}$.

Throughout the paper the letter $C$ denotes a generic positive constant which may vary in value from line to line.

2. Preliminaries and notations. As already mentioned, we will deal with the Schrödinger operator

$$
L=\operatorname{div}\left(A(x) \nabla_{x}\right)-\mu
$$

on the half-space $\mathbb{R}_{+}^{n}=\left\{x=\left(x_{1}, \ldots, x_{n}\right) \in \mathbb{R}^{n}: x_{n}>0\right\}, n \geq 3$. We put $L_{0}=\operatorname{div}\left(A(x) \nabla_{x}\right)$. We assume that the matrix $A(x)=\left(a_{i j}(x)\right)_{1 \leq i, j \leq n}$ is real, symmetric, and uniformly elliptic, i.e. there is $\lambda \geq 1$ such that $\lambda^{-1}\|\xi\|^{2} \leq\langle A(x) \xi, \xi\rangle \leq \lambda\|\xi\|^{2}$ for all $x \in \mathbb{R}_{+}^{n}$ and $\xi \in \mathbb{R}^{n}$. The coefficients $a_{i j}$ are $\lambda$-locally Lipschitz continuous. $\mu$ is a signed Radon measure.

We denote by $G_{0}$ the $L_{0}$-Green function on $\mathbb{R}_{+}^{n}$.

$G_{\Delta}$ denotes the Laplace-Green function on $\mathbb{R}_{+}^{n}$, which is given by

$$
G_{\Delta}(x, y)=w_{n}\left(\frac{1}{|x-y|^{n-2}}-\frac{1}{|x-\widetilde{y}|^{n-2}}\right),
$$

where $\widetilde{y}=\left(y^{\prime},-y_{n}\right)$ when $y=\left(y^{\prime}, y_{n}\right)$ with $y^{\prime} \in \mathbb{R}^{n-1}$ and $y_{n} \in \mathbb{R}$. Here $w_{n}=(2 \pi)^{-n / 2} \Gamma(n / 2-1)$ is the volume of the unit sphere $S^{n-1}$ in $\mathbb{R}^{n}$.

We recall that there is a constant $C=C(n, \lambda)>0$ such that

$$
C^{-1} G_{\Delta} \leq G_{0} \leq C G_{\Delta} \text {. }
$$


This is proved by integrating the corresponding semigroup densities with respect to time (see [8, Remark 2, p. 142]).

A function $u$ on $\mathbb{R}_{+}^{n}$ is called an $L$-solution if it is continuous and satisfies $L u=0$ in the distributional sense. We denote by $\mathcal{C}_{L}\left(\mathbb{R}_{+}^{n}\right)\left(\right.$ resp. $\left.\mathcal{C}_{\Delta}\left(\mathbb{R}_{+}^{n}\right)\right)$ the cone of positive $L$-solutions (resp. $\Delta$-solutions) on $\mathbb{R}_{+}^{n}$ continuously vanishing on the boundary.

\section{The class $\mathcal{K}$}

Definition 3.1. We say that a signed Radon measure $\mu$ on $\mathbb{R}_{+}^{n}$ is in the class $\mathcal{K}$ if it satisfies

$$
\sup _{x \in \mathbb{R}_{+}^{n}} \int_{\mathbb{R}_{+}^{n}} \frac{y_{n}}{x_{n}} G_{\Delta}(x, y)|\mu|(d y)<\infty .
$$

To study the class $\mathcal{K}$ we first give some interesting estimates of the Green function $G_{\Delta}$.

Lemma 3.2. There exists a constant $C>0$ such that for all $x, y \in \mathbb{R}_{+}^{n}$ :

(i) $\frac{1}{C} \frac{1}{|x-y|^{n-2}} \frac{x_{n} y_{n}}{|x-\widetilde{y}|^{2}} \leq G_{\Delta}(x, y) \leq \frac{C}{|x-y|^{n-2}} \frac{x_{n} y_{n}}{|x-\widetilde{y}|^{2}}$.

(ii) $\frac{1}{C} \frac{1}{|x-y|^{n-2}} \min \left(1, \frac{x_{n} y_{n}}{|x-y|^{2}}\right) \leq G_{\Delta}(x, y)$

$$
\leq \frac{C}{|x-y|^{n-2}} \min \left(1, \frac{x_{n} y_{n}}{|x-y|^{2}}\right) \text {. }
$$

(iii) $\frac{x_{n}}{y_{n}} G_{\Delta}(x, y) \leq \frac{C}{|x-y|^{n-2}}$.

Proof. We have

$$
\begin{aligned}
G_{\Delta}(x, y) & =w_{n}\left(\frac{1}{|x-y|^{n-2}}-\frac{1}{|x-\widetilde{y}|^{n-2}}\right) \\
& =\frac{w_{n}}{|x-y|^{n-2}}\left[1-\left(\frac{|x-y|^{2}}{|x-\widetilde{y}|^{2}}\right)^{(n-2) / 2}\right] \\
& =\frac{w_{n}}{|x-y|^{n-2}}\left[1-\left(1-\frac{4 x_{n} y_{n}}{|x-\widetilde{y}|^{2}}\right)^{(n-2) / 2}\right] .
\end{aligned}
$$

Using the inequalities

$$
\frac{t}{\alpha+2} \leq 1-(1-t)^{\alpha} \leq \frac{t}{\alpha+1} \quad \text { for } t \in(0,1) \text { and } \alpha>0
$$

we obtain

$$
\frac{8 w_{n}}{n+2} \frac{1}{|x-y|^{n-2}} \frac{x_{n} y_{n}}{|x-\widetilde{y}|^{2}} \leq G_{\Delta}(x, y) \leq \frac{8 w_{n}}{n} \frac{1}{|x-y|^{n-2}} \frac{x_{n} y_{n}}{|x-\widetilde{y}|^{2}},
$$

which proves (i). 
On the other hand,

$$
\frac{1}{5} \min \left(1, \frac{x_{n} y_{n}}{|x-y|^{2}}\right) \leq \frac{x_{n} y_{n}}{|x-\widetilde{y}|^{2}} \leq \min \left(1, \frac{x_{n} y_{n}}{|x-y|^{2}}\right) .
$$

Hence (ii) holds from (i) and (2).

We now prove (iii). From (ii), we have

$$
\begin{aligned}
\frac{x_{n}}{y_{n}} G_{\Delta}(x, y) & \leq \frac{C}{|x-y|^{n-2}} \min \left(\frac{x_{n}}{y_{n}}, \frac{x_{n}^{2}}{|x-y|^{2}}\right) \\
& \leq \frac{C}{|x-y|^{n-2}} \min \left(\frac{x_{n}}{y_{n}}, \frac{x_{n}^{2}}{\left|x_{n}-y_{n}\right|^{2}}\right) .
\end{aligned}
$$

Put $t=x_{n} / y_{n}>0$. We have

$$
\min \left(\frac{x_{n}}{y_{n}}, \frac{x_{n}^{2}}{\left|x_{n}-y_{n}\right|^{2}}\right)=\min \left(t,\left(\frac{t}{t-1}\right)^{2}\right) \leq 4 .
$$

Thus

$$
\frac{x_{n}}{y_{n}} G_{\Delta}(x, y) \leq \frac{4 C}{|x-y|^{n-2}}
$$

and (iii) is proved.

Proposition 3.3. For $\alpha \in \mathbb{R}$, the measure $y_{n}^{-\alpha} e^{-|y|} d y$ is in the class $\mathcal{K}$ if and only if $\alpha<2$.

Proof. We assume that $\alpha<2$ and we will prove

$$
\sup _{x \in \mathbb{R}_{+}^{n}} \int_{\mathbb{R}_{+}^{n}} \frac{y_{n}}{x_{n}} G_{\Delta}(x, y) y_{n}^{-\alpha} e^{-|y|} d y<\infty .
$$

If $\alpha<0$, then from Lemma 3.2(iii) we have

$$
\begin{aligned}
\int_{\mathbb{R}_{+}^{n}} \frac{y_{n}}{x_{n}} G_{\Delta} & (x, y) y_{n}^{-\alpha} e^{-|y|} d y \leq C \int_{\mathbb{R}_{+}^{n}} \frac{y_{n}^{-\alpha} e^{-|y|}}{|x-y|^{n-2}} d y \leq C \int_{\mathbb{R}_{+}^{n}} \frac{|y|^{-\alpha} e^{-|y|}}{|x-y|^{n-2}} d y \\
& \leq C \int_{\mathbb{R}_{+}^{n}} \frac{e^{-|y| / 2}}{|x-y|^{n-2}} d y=C\left(\int_{|x-y| \geq|y|} \ldots d y+\int_{|x-y| \leq|y|} \ldots d y\right) \\
& \leq C\left(\int_{\mathbb{R}^{n}} \frac{e^{-|y| / 2}}{|y|^{n-2}} d y+\int_{\mathbb{R}^{n}} \frac{e^{-|x-y| / 2}}{|x-y|^{n-2}} d y\right)=2 C w_{n} \int_{0}^{\infty} r e^{-r / 2} d r<\infty .
\end{aligned}
$$

If $0 \leq \alpha<2$, then from Lemma 3.2(ii), (iii), we have

$$
\begin{aligned}
& \int_{\mathbb{R}_{+}^{n}} \frac{y_{n}}{x_{n}} G_{\Delta}(x, y) y_{n}^{-\alpha} e^{-|y|} d y=\int_{|x-y| \geq y_{n}} \ldots d y+\int_{|x-y| \leq y_{n}} \ldots d y \\
& \leq C\left(\int_{|x-y| \geq y_{n}} \frac{y_{n}^{2-\alpha} e^{-|y|}}{|x-y|^{n}} d y+\int_{|x-y| \leq y_{n}} \frac{y_{n}^{-\alpha} e^{-|y|}}{|x-y|^{n-2}} d y\right)=C\left(I_{1}+I_{2}\right) .
\end{aligned}
$$


We estimate $I_{1}$ :

$$
\begin{aligned}
I_{1} & =\int_{|x-y| \geq y_{n}} \frac{y_{n}^{2-\alpha} e^{-|y|}}{|x-y|^{n}} d y \leq \int_{\mathbb{R}^{n}} \frac{e^{-|y|}}{|x-y|^{n-2+\alpha}} d y \\
& =\int_{|x-y| \geq|y|} \frac{e^{-|y|}}{|x-y|^{n-2+\alpha}} d y+\int_{|x-y| \leq|y|} \frac{e^{-|y|}}{|x-y|^{n-2+\alpha}} d y \\
& \leq \int_{\mathbb{R}^{n}} \frac{e^{-|y|}}{|y|^{n-2+\alpha}} d y+\int_{\mathbb{R}^{n}} \frac{e^{-|x-y|}}{|x-y|^{n-2+\alpha}} d y \\
& =2 w_{n} \int_{0}^{\infty} r^{1-\alpha} e^{-r} d r<\infty .
\end{aligned}
$$

Now we estimate $I_{2}$ :

$$
\begin{aligned}
I_{2} & =\int_{|x-y| \leq y_{n}} \frac{y_{n}^{-\alpha} e^{-|y|}}{|x-y|^{n-2}} d y \leq \int_{|x-y| \leq y_{n}} \frac{e^{-|x-y|}}{|x-y|^{n-2+\alpha}} d y \\
& \leq \int_{\mathbb{R}^{n}} \frac{e^{-|x-y|}}{|x-y|^{n-2+\alpha}} d y=w_{n} \int_{0}^{\infty} r^{1-\alpha} e^{-r} d r<\infty .
\end{aligned}
$$

Combining (3)-(5), we obtain

$$
\sup _{x \in \mathbb{R}_{+}^{n}} \int_{\mathbb{R}_{+}^{n}} \frac{y_{n}}{x_{n}} G_{\Delta}(x, y) y_{n}^{-\alpha} e^{-|y|} d y<\infty .
$$

Conversely, assume that $y_{n}^{-\alpha} e^{-|y|} d y \in \mathcal{K}$; we will prove $\alpha<2$. We have

$$
\sup _{x \in \mathbb{R}_{+}^{n}} \int_{\mathbb{R}_{+}^{n}} \frac{y_{n}}{x_{n}} G_{\Delta}(x, y) y_{n}^{-\alpha} e^{-|y|} d y<\infty,
$$

and from Lemma 3.2(ii) it follows that

$$
\sup _{x \in \mathbb{R}_{+}^{n}} \int_{\mathbb{R}_{+}^{n}} \min \left(\frac{y_{n}}{x_{n}}, \frac{y_{n}^{2}}{|x-y|^{2}}\right) \frac{y_{n}^{-\alpha} e^{-|y|}}{|x-y|^{n-2}} d y<\infty .
$$

This implies

$$
\sup _{x \in \mathbb{R}_{+}^{n}} \int_{\mathbb{R}_{+}^{n}} \min \left(\frac{y_{n}}{x_{n}}, \frac{y_{n}^{2}}{(|x|+|y|)^{2}}\right) \frac{y_{n}^{-\alpha} e^{-|y|}}{(|x|+|y|)^{n-2}} d y<\infty,
$$

which means

$$
\int_{\mathbb{R}_{+}^{n}} \frac{y_{n}^{2-\alpha} e^{-|y|}}{|y|^{n}} d y<\infty
$$


This yields

$$
\int_{0}^{\infty} y_{n}^{2-\alpha} e^{-y_{n}} \int_{\mathbb{R}^{n-1}} \frac{e^{-\left|y^{\prime}\right|}}{\left(\left|y^{\prime}\right|+y_{n}\right)^{n}} d y^{\prime} d y_{n}<\infty
$$

which means

$$
\int_{0}^{\infty} y_{n}^{2-\alpha} e^{-y_{n}} \int_{0}^{\infty} \frac{r^{n-2} e^{-r}}{\left(r+y_{n}\right)^{n}} d r d y_{n}<\infty
$$

Hence

$$
\int_{0}^{\infty} y_{n}^{2-\alpha} e^{-y_{n}} \int_{y_{n}}^{2 y_{n}} \frac{r^{n-2} e^{-r}}{\left(r+y_{n}\right)^{n}} d r d y_{n}<\infty
$$

and thus

$$
\int_{0}^{\infty} y_{n}^{1-\alpha} e^{-3 y_{n}} d y_{n}<\infty
$$

This necessarily implies that $\alpha<2$.

Now we will show that the class $\mathcal{K}$ is more general than the Kato class at infinity, $K_{n}^{\infty}$, considered by Zhao $[11,12]$ and Pinchover [6]. For the reader's convenience, we recall the definition of $K_{n}^{\infty}$.

Definition 3.4. We say that a Borel measurable function $V$ on $\mathbb{R}_{+}^{n}$, $n \geq 3$, is in the class $K_{n}^{\infty}$ if it satisfies

$$
\begin{aligned}
& \lim _{r \rightarrow 0} \sup _{x \in \mathbb{R}_{+}^{n}} \int_{(|x-y| \leq r) \cap \mathbb{R}_{+}^{n}} \frac{|V(y)|}{|x-y|^{n-2}} d y=0, \\
& \lim _{M \rightarrow+\infty} \sup _{x \in \mathbb{R}_{+}^{n}} \int_{(|y| \geq M) \cap \mathbb{R}_{+}^{n}} \frac{|V(y)|}{|x-y|^{n-2}} d y=0 .
\end{aligned}
$$

Note that if $V \in K_{n}^{\infty}$ then for all $M>0$,

$$
\int_{(|y| \leq M) \cap \mathbb{R}_{+}^{n}}|V(y)| d y<\infty .
$$

In particular $V \in L_{\text {loc }}^{1}\left(\mathbb{R}_{+}^{n}\right)$.

From [11], we have

Proposition 3.5. If $V \in K_{n}^{\infty}$, then $V$ is Green bounded, i.e.

$$
\sup _{x \in \mathbb{R}_{+}^{n}} \int_{\mathbb{R}_{+}^{n}} \frac{|V(y)|}{|x-y|^{n-2}} d y<\infty .
$$

Proposition 3.6. The class $\mathcal{K}$ strictly contains the class of Green bounded potentials. 
Proof. Since by Lemma 3.2(iii),

$$
\frac{y_{n}}{x_{n}} G_{\Delta}(x, y) \leq \frac{1}{|x-y|^{n-2}},
$$

it follows from Proposition 3.5 that when $V$ is in $K_{n}^{\infty}, V(x) d x$ is in $\mathcal{K}$. Moreover for $1 \leq \alpha<2$ and $M>0$ we have

$$
\int_{(|y| \leq M) \cap \mathbb{R}_{+}^{n}} y_{n}^{-\alpha} e^{-|y|} d y \geq e^{-M} \int_{(|y| \leq M) \cap \mathbb{R}_{+}^{n}} y_{n}^{-\alpha} d y=+\infty .
$$

This implies that $y_{n}^{-\alpha} e^{-|y|} \notin K_{n}^{\infty}$. The conclusion follows from Proposition 3.3 .

4. The $L$-Green function. In this section we study the existence of the $L$-Green function $G$ and its comparablity to $G_{\Delta}$ when $\mu$ is in the class $\mathcal{K}$.

Following the classical potential theory, a Borel measurable function $G$ : $\left.\left.\mathbb{R}_{+}^{n} \times \mathbb{R}_{+}^{n} \rightarrow\right] 0,+\infty\right]$ is called a Green function for the Schrödinger operator $L=\Delta-\mu$ if for all $y \in \mathbb{R}_{+}^{n}, L G(\cdot, y)=-\varepsilon_{y}$ on $\mathbb{R}_{+}^{n}$ in the distributional sense, where $\varepsilon_{y}$ is the Dirac measure at $y$ and $G(\cdot, y)$ vanishes outside a polar set on $\partial \mathbb{R}_{+}^{n}$. To prove our main result we shall first prove the following 3G-Theorem which was established in the two-dimensional case in [9].

TheOREM 4.3 (3G-Theorem). There exists a positive constant $C_{0}$ such that for $x, y, z \in \mathbb{R}_{+}^{n}$, we have

$$
\frac{G_{0}(x, z) G_{0}(z, y)}{G_{0}(x, y)} \leq C_{0}\left(\frac{z_{n}}{x_{n}} G_{0}(x, z)+\frac{z_{n}}{y_{n}} G_{0}(z, y)\right) .
$$

Proof. By (1) and Lemma 3.2(i), we have

$$
\frac{1}{C} N(x, y) \leq G_{0}(x, y) \leq C N(x, y),
$$

where

$$
N(x, y)=\frac{1}{|x-y|^{n-2}} \frac{x_{n} y_{n}}{|x-\widetilde{y}|^{2}} .
$$

Thus to prove the theorem it suffices to prove the inequality

$$
\frac{N(x, z) N(z, y)}{N(x, y)} \leq C\left(\frac{z_{n}}{x_{n}} N(x, z)+\frac{z_{n}}{y_{n}} N(z, y)\right),
$$

which is equivalent to

$$
|x-y|^{n-2}|x-\widetilde{y}|^{2} \leq C\left(|x-z|^{n-2}|x-\widetilde{z}|^{2}+|z-y|^{n-2}|z-\widetilde{y}|^{2}\right) .
$$

By symmetry we may assume $|x-z| \leq|z-y|$. We have

$$
|x-y|^{n-2} \leq(|x-z|+|z-y|)^{n-2} \leq 2^{n-2}|z-y|^{n-2},
$$

and

$$
|x-\widetilde{y}|^{2} \leq(|x-z|+|z-\widetilde{y}|)^{2}=2^{2}|z-\widetilde{y}|^{2},
$$


which yields

$$
|x-y|^{n-2}|x-\widetilde{y}|^{2} \leq 2^{n}|z-y|^{n-2}|z-\widetilde{y}|^{2},
$$

and (6) follows with $C=2^{n}$.

Now we are ready to prove the main result of this section.

Theorem 4.4. Let $\mu \in \mathcal{K}$ with $4 C_{0}\|\mu\|<1$. Then the $L$-Green function $G$ exists and is comparable to $G_{\Delta}$, i.e. there is a constant $C>0$ such that

$$
C^{-1} G_{\Delta} \leq G \leq C G_{\Delta} .
$$

Proof. From the 3G-Theorem, we have

$$
\begin{aligned}
\int_{\mathbb{R}_{+}^{n}} G_{0}(x, z) G_{0}(z, y)|\mu|(d z) & \\
& \leq C_{0} \int_{\mathbb{R}_{+}^{n}}\left(\frac{z_{n}}{x_{n}} G_{0}(x, z)+\frac{z_{n}}{y_{n}} G_{0}(z, y)\right)|\mu|(d z) G_{0}(x, y) \\
& \leq 2 C_{0}\|\mu\| G_{0}(x, y) .
\end{aligned}
$$

Since $G_{0}<\infty$ outside the diagonal and $|\mu|\{x\}=0$ for all $x \in \mathbb{R}_{+}^{n}$, it follows that for $x \neq y \in \mathbb{R}_{+}^{n}$ and $m \in \mathbb{N}$, we may define

$$
G_{0}^{* m+1}(x, y)=G_{0}^{* m} * G_{0}(x, y) \equiv \int_{\mathbb{R}_{+}^{n}} G_{0}^{* m}(x, z) G_{0}(z, y) \mu(d z)
$$

with $G_{0}^{* 0}=G_{0}$. By induction we obtain

$$
\left|G_{0}^{* m}(x, y)\right| \leq\left(2 C_{0}\|\mu\|\right)^{m} G_{0}(x, y) .
$$

Since $2 C_{0}\|\mu\|<1$, it follows that

$$
\sum_{m \geq 0}\left|G_{0}^{* m}(x, y)\right| \leq \frac{1}{1-2 C_{0}\|\mu\|} G_{0}(x, y) .
$$

We then define $G$ by

$$
G(x, y)= \begin{cases}+\infty & \text { if } x=y \\ \sum_{m \geq 0}(-1)^{m} G_{0}^{* m}(x, y) & \text { if } x \neq y .\end{cases}
$$

Clearly, since $G_{0}$ vanishes on $\partial \mathbb{R}_{+}^{n}$, it follows from the previous inequality that $G$ has the same property. Moreover, we have

$$
G_{0}(\cdot, y)=G(\cdot, y)+\int_{\mathbb{R}_{+}^{n}} G_{0}(\cdot, z) G(z, y) \mu(d z) \quad \text { on } \mathbb{R}_{+}^{n} \backslash\{y\} .
$$

This resolvent equation implies the equality

$$
L G(\cdot, y)=-\varepsilon_{y}
$$


in the distributional sense. Thus $G$ is the $L$-Green function on $\mathbb{R}_{+}^{n}$. Moreover, for all $x \neq y$, we have

$$
G(x, y)-G_{0}(x, y)=\sum_{m \geq 1}(-1)^{m} G_{0}^{* m}(x, y) .
$$

Hence, for all $x \neq y$, we have

$$
\left|G(x, y)-G_{0}(x, y)\right| \leq \sum_{m \geq 1}\left(2 C_{0}\|\mu\|\right)^{m} G_{0}(x, y)=\frac{2 C_{0}\|\mu\|}{1-2 C_{0}\|\mu\|} G_{0}(x, y) .
$$

By recalling that $G=G_{0}=+\infty$ on the diagonal and that $4 C_{0}\|\mu\|<1$, we get

$$
\frac{1-4 C_{0}\|\mu\|}{1-2 C_{0}\|\mu\|} G_{0} \leq G \leq \frac{1}{1-2 C_{0}\|\mu\|} G_{0} .
$$

Since by (1), $G_{0}$ is comparable to $G_{\Delta}$, the proof is complete.

5. The structure of $\mathcal{C}_{L}\left(\mathbb{R}_{+}^{n}\right)$. The comparison theorem implies that when $\mu$ is in $\mathcal{K}$ with $4 C_{0}\|\mu\|<1$, the $L$-Green function behaves like the $\Delta$-Green function in particular at infinity and near the boundary $\left\{x_{n}=0\right\}$. This fact enables us to prove that the Martin boundaries with respect to $L$ and $\Delta$ are homeomorphic. The proof follows the idea of Theorem 2.3 in [7]. Using this observation and potential-theoretic arguments we will prove that the set $\mathcal{C}_{L}\left(\mathbb{R}_{+}^{n}\right)$ of all positive $L$-solutions on $\mathbb{R}_{+}^{n}$ continuously vanishing on the boundary $\left\{x_{n}=0\right\}$ is a one-dimensional cone. This kind of result fails to hold even in simple cases such as $L=\Delta-c$, where $c$ is a nonzero real constant. In fact our main result is the following.

TheOREM 5.1. Let $\mu$ be in $\mathcal{K}$ with $4 C_{0}\|\mu\|<1$. Then the set $\mathcal{C}_{L}\left(\mathbb{R}_{+}^{n}\right)$ is a one-dimensional cone generated by a function equivalent to $x_{n}$.

Before we prove our main result we shall determine the Martin boundary with respect to $\Delta$ on the half-space. We first briefly recall the notion of the Martin boundary introduced by Martin in [5].

Let $\Omega$ be a domain in $\mathbb{R}^{n}$ and let $x_{0}$ be a fixed reference point in $\Omega$. Recall that a sequence $\left(y_{m}\right)$ in $\Omega$ is called fundamental if it has no accumulation point and for any $x \in \Omega$, the $\operatorname{limit}_{\lim _{m}} G_{\Delta}\left(x, y_{m}\right) / G_{\Delta}\left(x_{0}, y_{m}\right)$ exists. Here $G_{\Delta}$ denotes the Laplace-Green function on $\Omega$. Two fundamental sequences are called equivalent if the corresponding limits are the same. The class of all fundamental sequences equivalent to a given one determines an ideal boundary element of $\Omega$. The set of ideal boundary elements of $\Omega$ is called the Martin boundary of $\Omega$ corresponding to $\Delta$. For any Martin boundary element $z=\left(y_{m}\right)$, we write

$$
K_{\Delta}(x, z)=\lim _{m} \frac{G_{\Delta}\left(x, y_{m}\right)}{G_{\Delta}\left(x_{0}, y_{m}\right)},
$$


which is a positive $\Delta$-harmonic function of $x$ in $\Omega . K_{\Delta}(\cdot, z)$ will be called the $\Delta$-Martin kernel with pole $z$ normalized at $x_{0}$.

We will study the case of $\Omega=\mathbb{R}_{+}^{n}$. Let $\overline{\mathbb{R}^{n}}$ be the compactification of $\mathbb{R}^{n}$ which adjoins the sphere at infinity $S_{\infty}^{n-1}$. Specifically $S_{\infty}^{n-1}$ is a copy of the unit sphere $S^{n-1}$ in $\mathbb{R}^{n}$. Let $\left(y_{m}\right)_{m}$ be a sequence in $\mathbb{R}_{+}^{n}$ which has no accumulation point. By compactness of $\overline{\mathbb{R}^{n}}$ it has a subsequence still denoted $\left(y_{m}\right)_{m}$ which tends to $z \in \partial \mathbb{R}_{+}^{n}$ or to $e \in S_{+(\infty)}^{n-1}$ as $m \rightarrow \infty$, where $S_{+(\infty)}^{n-1}$ is a copy of the half-sphere $S_{+}^{n-1}=\left\{e \in S^{n-1}: e_{n}>0\right\}$. We identify $S_{+(\infty)}^{n-1}$ with $S_{+}^{n-1}$.

Recall that

$$
G_{\Delta}(x, y)=\frac{w_{n}}{|x-y|^{n-2}}\left[1-\left(1-\frac{4 x_{n} y_{n}}{|x-\widetilde{y}|^{2}}\right)^{\frac{n-2}{2}}\right] .
$$

For $z \in \partial \mathbb{R}_{+}^{n}=\left\{x_{n}=0\right\}$, we have

$$
\begin{aligned}
\lim _{y \rightarrow z, y \in \mathbb{R}_{+}^{n}} \frac{G_{\Delta}(x, y)}{G_{\Delta}\left(x_{0}, y\right)} & =\lim _{y \rightarrow z, y \in \mathbb{R}_{+}^{n}} \frac{|x-y|^{2-n}}{\left|x_{0}-y\right|^{2-n}} \frac{x_{n}}{x_{0, n}} \frac{\left|x_{0}-\widetilde{y}\right|^{2}}{|x-\widetilde{y}|^{2}} \\
& =\frac{x_{n}}{x_{0, n}} \frac{\left|x_{0}-z\right|^{n}}{|x-z|^{n}} .
\end{aligned}
$$

This shows that any $z \in \partial \mathbb{R}_{+}^{n}$ is a Martin boundary element and

$$
K_{\Delta}(x, z)=\frac{x_{n}}{x_{0, n}} \frac{\left|x_{0}-z\right|^{n}}{|x-z|^{n}} .
$$

Moreover we have

$$
\lim _{|y| \rightarrow \infty, y \in \mathbb{R}_{+}^{n}} \frac{G_{\Delta}(x, y)}{G_{\Delta}\left(x_{0}, y\right)}=\lim _{|y| \rightarrow \infty, y \in \mathbb{R}_{+}^{n}} \frac{|x-y|^{2-n}}{\left|x_{0}-y\right|^{2-n}} \frac{x_{n}}{x_{0, n}} \frac{\left|x_{0}-\widetilde{y}\right|^{2}}{|x-\widetilde{y}|^{2}}=\frac{x_{n}}{x_{0, n}},
$$

which shows that any $e \in S_{+}^{n-1}$ is a Martin boundary element and $K_{\Delta}(x, e)$ $=x_{n} / x_{0, n}$.

Thus the Martin boundary with respect to $\Delta$ is identical to $\partial \mathbb{R}_{+}^{n} \cup S_{+}^{n-1}$ and by the Martin representation theorem, for every positive $\Delta$-solution $u$ on $\mathbb{R}_{+}^{n}$, there are two positive Borel measures $\sigma$ on $\partial \mathbb{R}_{+}^{n}$ and $\nu$ on $S_{+}^{n-1}$ such that

$$
\begin{aligned}
u(x) & =\int_{\partial \mathbb{R}_{+}^{n}} K_{\Delta}(x, z) d \sigma(z)+\int_{S_{+}^{n-1}} K_{\Delta}(x, e) d \nu(e) \\
& =\frac{x_{n}}{x_{0, n}} \int_{\partial \mathbb{R}_{+}^{n}} \frac{\left|x_{0}-z\right|^{n}}{|x-z|^{n}} d \sigma(z)+\alpha \frac{x_{n}}{x_{0, n}} .
\end{aligned}
$$

Obviously the first part in the right member of this representation tends to zero at infinity. Hence when $u$ tends to zero as $x_{n} \rightarrow 0^{+}$, we get $u(x)=$ $\alpha x_{n} / x_{0, n}$. This shows that $\mathcal{C}_{\Delta}\left(\mathbb{R}_{+}^{n}\right)$ is a one-dimensional cone generated by the function $u_{0}(x)=x_{n}$. 
Proof of Theorem 5.1. Since by Theorem 4.4, $G$ and $G_{\Delta}$ are comparable, the Martin boundary with respect to $L$ is homeomorphic to the Martin boundary with respect to $\Delta$ (Theorem 2.3 in [7]), and so it is identical to $\partial \mathbb{R}_{+}^{n} \cup S_{+}^{n-1}$. Moreover, for all $x \in \mathbb{R}_{+}^{n}$ and $z \in \partial \mathbb{R}_{+}^{n} \cup S_{+}^{n-1}$,

$$
C^{-2} K_{\Delta}(x, z) \leq K_{L}(x, z) \leq C^{2} K_{\Delta}(x, z),
$$

where $C$ is the constant which occurs in Theorem 4.4.

Now let $v \in \mathcal{C}_{L}\left(\mathbb{R}_{+}^{n}\right)$. By the Martin representation theorem, there are two positive Borel measures $\sigma$ on $\partial \mathbb{R}_{+}^{n}$ and $\nu$ on $S_{+}^{n-1}$ such that

$$
v(x)=\int_{\partial \mathbb{R}_{+}^{n}} K_{L}(x, z) d \sigma(z)+\int_{S_{+}^{n-1}} K_{L}(x, e) d \nu(e) .
$$

From (7), it follows that the function

$$
\begin{aligned}
u(x) & =\int_{\partial \mathbb{R}_{+}^{n}} K_{\Delta}(x, z) d \sigma(z)+\int_{S_{+}^{n-1}} K_{\Delta}(x, e) d \nu(e) \\
& =\int_{\partial \mathbb{R}_{+}^{n}} K_{\Delta}(x, z) d \sigma(z)+\alpha \frac{x_{n}}{x_{0, n}}
\end{aligned}
$$

is in $\mathcal{C}_{\Delta}\left(\mathbb{R}_{+}^{n}\right)$. Hence $\sigma \equiv 0$ and $u(x)=\alpha x_{n} / x_{0, n}$. This implies that

$$
v(x)=\int_{S_{+}^{n-1}} K_{L}(x, e) d \nu(e) .
$$

On the other hand, from (7) we have, for all $e \in S_{+}^{n-1}$,

$$
C^{-2} \frac{x_{n}}{x_{0, n}} \leq K_{L}(x, e) \leq C^{2} \frac{x_{n}}{x_{0, n}} .
$$

It follows that, for all $e, \widetilde{e} \in S_{+}^{n-1}$,

$$
C^{-4} K_{L}(\cdot, \widetilde{e}) \leq K_{L}(\cdot, e) \leq C^{4} K_{L}(\cdot, \widetilde{e}) .
$$

Since $K_{L}(\cdot, e)$ is a minimal positive $L$-solution and $K_{L}\left(x_{0}, e\right)=K_{L}\left(x_{0}, \widetilde{e}\right)$ $=1$, we deduce that for all $e, \widetilde{e} \in S_{+}^{n-1}$,

$$
K_{L}(\cdot, e)=K_{L}(\cdot, \widetilde{e})
$$

Hence we deduce from (8) and (9) that $v=\alpha K_{L}(\cdot, e)$ for some $\alpha \geq 0$. This shows that $\mathcal{C}_{L}\left(\mathbb{R}_{+}^{n}\right)$ is a one-dimensional cone generated by $K_{L}(\cdot, e)$. Moreover by $(7), K_{L}(\cdot, e)$ is equivalent to $x_{n}$, which completes the proof.

\section{References}

[1] A. Ancona, Comparaison des mesures harmoniques et des fonctions de Green pour des opérateurs elliptiques sur un domaine lipschitzien, C. R. Acad. Sci. Paris 294 (1982), 505-508. 
[2] M. Cranston, E. Fabes and Z. Zhao, Conditional gauge and potential theory for the Schrödinger operator, Trans. Amer. Math. Soc. 307 (1988), 171-194.

[3] I. W. Herbst and Z. Zhao, Green functions for the Schrödinger equation with short range potentials, Duke Math. J. 59 (1989), 475-519.

[4] H. Hueber and M. Sieveking, Uniform bounds for quotients of Green functions on $C^{1,1}$ domains, Ann. Inst. Fourier (Grenoble) 32 (1982), no. 1, 105-117.

[5] R. S. Martin, Minimal positive harmonic functions, Trans. Amer. Math. Soc. 49 (1941), 137-172.

[6] Y. Pinchover, On the equivalence of Green functions of second order elliptic equations in $\mathbb{R}^{n}$, Differential and Integral Equations 5 (1992), 481-493.

[7] -, On positive solutions of second order elliptic equations. Stability results and classification, Duke Math. J. 57 (1988), 955-980.

[8] L. Riahi, Green function bounds and parabolic potentials on a half-space, Potential Anal. 15 (2001), 133-150.

[9] M. Selmi, Critère de comparaison des noyaux de Green sur certains domaines de $\mathbb{R}^{2}$, Rev. Roumaine Math. Pures Appl. 42 (1997), 319-337.

[10] Z. Zhao, Green function for Schrödinger operator and conditional Feynman-Kac gauge, J. Math. Anal. Appl. 116 (1986), 309-334.

[11] - On the existence of positive solutions of nonlinear elliptic equations. A probabilistic potential theory approach, Duke Math. J. 69 (1993), 247-258.

[12] —, Subcriticality, positivity, and gaugeability of the Schrödinger operator, Bull. Amer. Math. Soc. 23 (1990), 513-517.

Department of Mathematics

Faculty of Sciences of Tunis

Campus Universitaire

1060 Tunis, Tunisia

E-mail: lotfi.riahi@fst.rnu.tn 